\title{
Unidad de Agudos: tratamientos antipsicóticos y de larga duración y criterios de calidad para la reducción de riesgos y mortalidad
}

\author{
Acute Unit: antipsychotic and long-term
criteria to reduce risks and mortality \\ Vicente Tordera-Tordera* \\ Unidad de Hospitalización Psiquiátrica, Hospital Lluís Alcanyís de Xátiva, Valencia, España
}

\section{Resumen}

Los antipsicóticos son el tratamiento de elección para el paciente con esquizofrenia. Tanto los antipsicóticos típicos ō de primera generación como los atípicos o de segunda generación son eficaces en los síntomas positivos que presentáa el paciente, aunque sus beneficios no son inocuos. Los de primera generación producen más efectos extrapiramidales y đạiscinesia tardía, y los de segunda generación provocan mayor ganancia de peso y posibles efectos cardiovasculares. En ēeste artículo se revisa brevemente las características de estos fármacos y el posible aumento del riesgo de mortalidad a corto y largo término relacionada con su uso frente a los riesgos de no tratar. Por otro lado, la aparición de los antipsicóticos inyectables de larga duración supone un cambio en los hábitos de prescripción. Su implementación y uso desde el inicio de la enfermedad, y su influencia en los criterios de calidad en nuestra labor asistencial en la sala de agudos nos abocan a nuevos retos en nuestra práctica diaria.

Palabras claves: Psicofármacos. Antipsicóticos. Mortalidad. Criterios de calidad.

\begin{abstract}
Antipsychotics are the treatment of choice for the patient with schizophrenia. Both typical or first-generation antipsychoties, as well as atypical or second-generation antipsychotics, are specific to the positive symptoms that the patient presents, although their benefits are not harmless. Those of the first generation produce more extrapyramidal effects and tardive dyskinesia those of the second generation cause greater weight gain and possible cardiovascular effects. This article briefly reviews the characteristics of these drugs and the possible increase in the risk of short-and long-term mortality related to their use versus the risks of no treatment. On the other hand, the appearance of long-lasting injectable antipsychotics implies a change in prescription habits. Its implementation and use since the beginning of the disease, as well as its influence on the quality criteria in our assisting job within the acute unit, offer us new challenges in our daily practice.

Key words: Psychopharmaceuticals. Antipsychotics. Mortality. Quality criteria.

Correspondencia:

*Vicente Tordera-Tordera

E-mail: tordera2@ hotmail.com

Fecha de recepción: 12-09-2019

Fecha de aceptación: 01-11-2019

DOI: 10.24875/RPSIC.M19000002

Disponible en internet: 09-12-2019

Revis Psicofarmacol. 2019;5(1):25-33

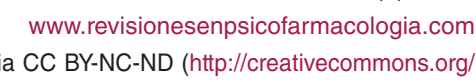
licenses/by-nc-nd/4.0/)
\end{abstract}




\section{Introducción}

Según el informe de la Organización Mundial de la Salud del año 2013, los problemas mentales en Europa afectan al $25 \%$ de la población cada año ${ }^{1}$, y la previsión es que serán la segunda causa de incapacidad en el año $2020^{2}$.

La psicofarmacología es una disciplina compleja, cuyo objetivo es modular las emociones humanas, el comportamiento y otros procesos cognitivos complejos.

En sus inicios la clorpromazina representó, en la década de 1950, el inicio de la psicofarmacología moderna ${ }^{3}$. En esa época, además de la clorpromazina, que fue el primer antipsicótico, se desarrollaron otros fármacos como los ansiolíticos, los inhibidores de la monoaminooxidasa y los antidepresivos tricícli$\cos ^{3}$. No fue hasta la década de 1990 cuando se produjo un importante avance en la psicofarmacología con la aparición de los primeros antipsicóticos atípicos (risperidona, olanzapina, quetiapina), con menos efectos extrapiramidales, si bien posteriormente se observó que no se mejoraba el perfil de seguridad $^{3}$.

Esta evolución en la psicofarmacología influyó enormemente en la asistencia clínica de los pacientes, en la metodología de la investigación clínica (principalmente de instrumentos de medida), en la reducción del número de pacientes ingresados y en los días de hospitalización ${ }^{4}$. A pesar de ello es evidente que siguen existiendo problemas en el tratamiento de estos pacientes; se necesitan fármacos más eficaces, más rápidos y específicos y con mínimos efectos secundarios ${ }^{4}$.

\section{Antipsicóticos (neurolépticos)}

Empleados principalmente como método de control de las crisis psicóticas, este grupo de psicofármacos eran antiguamente denominados tranquilizantes mayores debido al nivel de sedación que causaban inicialmente.

Todos los antipsicóticos tienen un mecanismo de acción común entre ellos que es actuar como antagonistas de los receptores dopaminérgicos cerebrales ${ }^{5}$. Algunos presentan otras propiedades adicionales, como el antagonismo de los receptores de serotonina-dopamina y/o el agonismo parcial dopaminérgico. En función de este mecanismo de acción, se clasifican como antipsicóticos típicos (convencionales o de primera generación) y antipsicóticos atípicos (o de segunda generación) $)^{5}$.

\section{Antipsicóticos de primera generación típicos}

Además de la clorpromazina, existe una gran cāntidad de fármacos antipsicóticos clásicos que se clâsifican en diferentes familias y cuya actividad se basă en el bloqueo de los receptores de dopamina D2. Aparte de su eficacia sobre los síntomas positivos de la esquizofrenia y trastornos psicóticos, pueden provocar importantes efectos adversos que en muchas ocassiones obligan a abandonar la medicación ${ }^{4,6}$.

Uno de los efectos adversos que presentan losantipsicóticos y que tiene mayor incidencia en los pacientes es la aparición de efectos motores extrapiramidales, que pueden ser agudos o a largo plazo, siempre acompañados de síntomas motores objetivos y mentales subjetivos. Este efecto se considera la prinẹppal causa de abandono del tratamiento ${ }^{4,6}$.

Entre las reacciones agudas se puede observar a tisia (en el $25 \%$ de los pacientes), que puede aparécer a las pocas horas o días de iniciar el tratamiente, la distonía aguda y los síntomas parkinsonianos, que ęcurren en el $50 \%$ de los pacientes ${ }^{4}$.

También pueden producir reacciones extrapiramidales tardías como la discinesia tardía, que es dependiente del tiempo y de la dosis ${ }^{4,6}$. El riesgo estimado de discinesia tardía con los antipsicóticos de primera generación es del 3-5\% por año de exposición (al menos durante los primeros cinco años) y es menorçon los de segunda generación, siendo estos los fármạcos de primera línea como tratamiento de mantenimiênto para reducir el riesgo de discinesia tardía? Otros síntomas asociados al tratamiento crónico son la distônía tardía y la acatisia tardía ${ }^{4}$. En resumen, los antipsicóticos típicos, en general, interfieren con las funciones motoras normales además de producir alteraciōnes endocrinológicas ${ }^{5}$.

En general, los antipsicóticos clásicos son pocoseficaces en el manejo de los síntomas negativos que presentan estos pacientes. Su aparición y uso coh̆stituyen un avance innegable, pero en la actualida encuentran claramente superados por los antipsič́ticos atípicos.

Se han realizado diversos estudios y metaanáfisis comparando la eficacia y la tolerabilidad de los antipsicóticos de primera generación frente a los de segunda generación. Uno de ellos incluyó 149 pacieñtes (69 con antipsicóticos de primera generación y $80^{\circ} \mathrm{pa}$ cientes con los de segunda generación) tratados durante 24 semanas y en los que se valoró su efêcto en la calidad de vida mediante la escala SF-36. La 
Tabla 1. Afinidad receptora de los receptores de los atípicos

\begin{tabular}{|c|c|c|c|c|c|c|c|c|c|c|c|c|}
\hline \multicolumn{13}{|c|}{ Perfil receptora de los principales fármacos antipsicóticos, afinidad por receptores in vitro: valores Ki (nM) } \\
\hline & D1 & $\mathbf{D 2}$ & D3 & D4 & 5-HT1A & 5-HT2A & 5-HT2C & 5-HT3 & a1 & a2 & Musc & Hist \\
\hline \multicolumn{13}{|c|}{ Antipsicóticos típicos } \\
\hline Hal & 14,9 & 0,8 & - & 2,5 & 2548 & 28,3 & 1.514 & 49.495 & 7,3 & 1.584 & 575 & $>$ \\
\hline Flu & 0,7 & 0,5 & - & - & 1903 & 5,7 & 161 & $>5.000$ & 8,5 & 119 & 199 & 19 \\
\hline \multicolumn{13}{|c|}{ Antipsicóticos atípicos } \\
\hline $\mathrm{Clz}$ & 53,5 & 44,6 & - & 21,5 & 712 & 4,0 & 5,1 & 44,1 & 3,7 & 50,7 & 1,0 & 16 \\
\hline Ser & 11,5 & 0,6 & - & 10,5 & 2.152 & 0,2 & 0,5 & 9.404 & 1,4 & 277 & 261 & \\
\hline Ris & 20,6 & 0,4 & - & 12,5 & 767 & 0,4 & 6,4 & $>10.000$ & 0,7 & 1,8 & 679 & \\
\hline Olz & 10,3 & 2,1 & - & 16,5 & 1.173 & 1,9 & 2,8 & 40,0 & 7,3 & 143 & 2,1 & 5 \\
\hline Zip & 9,5 & 2,8 & - & 14,0 & 7,0 & 0,3 & 0,6 & - & 1,9 & - & $>10.000$ & \\
\hline Que & 387 & 69 & - & $>500$ & 1.655 & 82 & 1.514 & 9.899 & 4,5 & 1.108 & 1.514 & 20 \\
\hline Ami & $<$ & 4,4 & 5,1 & $>$ & $>$ & 500 & 500 & $>$ & 500 & $>$ & $>$ & \\
\hline Ari & 265 & 0,45 & 0,8 & 44 & 4,4 & 3,4 & 15 & 150 & 47 & 791 & $>$ & \\
\hline $\mathrm{Pal}$ & 25 & 0,4 & - & 12,5 & 767 & 400 & 1,4 & $>10.000$ & 7 & 5 & 600 & \\
\hline
\end{tabular}

puntuación de calidad de vida medida con la escala SF-36 fue significativamente mayor en los pacientes tratados con los de segunda generación comparado con los de primera ${ }^{8}$.

Un reciente metaanálisis para valorar el riesgo de discinesia tardía en pacientes tratados con antipsicóticos incluyó 57 estudios clínicos representando 32 grupos tratados con antipsicóticos de primera generación y 86 grupos tratados con los de segunda generación. Los resultados mostraron que la incidencia anual de discinesia tardía en el grupo tratado con primera generación fue del $6,5 \%$, mientras que en el grupo de segunda generación fue del 2,6\%, y tanto el riesgo de discinesia tardía como los índices anualizados fueron significativamente menores con los de segunda que con los de primera generación ${ }^{9}$.

\section{Antipsicóticos atípicos}

Los antipsicóticos atípicos se sintetizaron para ser más eficaces sobre los síntomas negativos, mejorar la tolerabilidad y provocar menos extrapiramidalismo ${ }^{4}$. En general se ha demostrado que reducen la confusión y la agitación en los pacientes psicóticos, mejorando además la actividad psicomotora y la afectación conductual y cognitiva ${ }^{4}$.
Los antipsicóticos pueden actuar sobre distintos receptores, lo que está relacionado con su eficacia y también con la aparición de efectos secundarios ${ }^{4}$ (Tablas 1y 2 ).

Los atípicos sí tienen la capacidad de bloqueapilos receptores serotoninérgicos 5-HT2A, lo que produciría menos efectos extrapiramidales. Con algunos antipsicóticos se ha observado sedación, somnolencia yัyaumento de peso, producidos como consecuencia del bloqueo de los receptores histaminérgicos $(\mathrm{H} 1)$.

El aumento de peso es una cuestión a tener en cưenta, ya que aumenta el riesgo cardiovascular ${ }^{4,7}$ y së ha relacionado además con la falta de cumplimiento. Ëste incremento de peso es distinto en función del atípico utilizado, aunque se ha observado que el sexo femenino es un predictor de aumento ponderal después de un tratamiento prolongado con atípicos ${ }^{12}$. Un estúdio danés sobre el riesgo de diabetes con antipsicóticos comparó a casi 350.000 pacientes tratados con antipsicóticos y casi 1,5 millones de individuos sin tratamiento y concluyó que el riesgo relativo de aumentar el peso y posteriormente presentar diabetes erâ de 1,53 en los pacientes que utilizaron los de primera generación y de 1,32 en los que utilizaron los de segun$\mathrm{da}^{13}$. Además, también concluyeron que el riesggo aumenta en función de la duración del tratamiento, así como con el uso de polifarmacia. 
Tabla 2. Intensidad de la afinidad receptora de los principales antipsicóticos

\begin{tabular}{|c|c|c|c|c|c|c|}
\hline Receptor & Ziprasidona & Risperidona & Olanzapina & Quetiapina & Clozapina & Aripipraz \\
\hline D2 & ++++ & ++++ & ++ & + & + & ++++ \\
\hline 5-HT2A & +++++ & +++++ & ++++ & + & ++++ & ++++ \\
\hline 5-HT2C & +++++ & ++++ & ++++ & - & ++ & ++ \\
\hline 5-HT1A & ++++ & + & - & + & + & ++++ \\
\hline 5-HT1D & ++++ & + & + & - & - & - \\
\hline Alfa1-adrenérgico & ++ & ++++ & ++ & ++ & ++++ & ++ \\
\hline M1 & - & - & ++++ & ++ & ++++ & - \\
\hline $\mathrm{H} 1$ & ++ & ++ & ++++ & ++++ & ++++ & ++ \\
\hline
\end{tabular}

Adaptada de Landa Contreras, et al., 2011"1.

Tabla 3. Efecto terapéutico y efectos adversos de los antipsicóticos en función del receptor bloqueado

\begin{tabular}{|c|c|c|}
\hline Receptor bloqueado & Efecto terapéutico & Efecto adverso \\
\hline $\mathrm{D} 2$ & Mejora síntomas psicóticos positivos & $\begin{array}{l}\text { Extrapiramidalismo } \\
\text { Efectos endocrinológicos }\end{array}$ \\
\hline Colinérgico muscarínico (M) & Reducción síntomas extrapiramidales & $\begin{array}{l}\text { Visión borrosa } \\
\text { Hipertensión intraocular } \\
\text { Sequedad de boca } \\
\text { Taquicardia sinusal } \\
\text { Estreñimiento } \\
\text { Retención urinaria } \\
\text { Alteraciones de la memoria }\end{array}$ \\
\hline $5-\mathrm{HT} 2$ & $\begin{array}{l}\text { Mejoría de los síntomas negativos } \\
\text { Reducción extrapiramidalismo }\end{array}$ & $\begin{array}{l}\text { Hipotensión ¿? } \\
\text { Somnolencia ¿? }\end{array}$ \\
\hline $\mathrm{H} 1$ & Sedación & $\begin{array}{l}\text { Sedación/somnolencia } \\
\text { Aumento de peso } \\
\text { Efectos depresores centrales }\end{array}$ \\
\hline Alfa1-adrenérgico & Ansiolisis & $\begin{array}{l}\text { Hipotensión ortostática } \\
\text { Taquicardia }\end{array}$ \\
\hline
\end{tabular}

Adaptada de Álamo, et al., 20044.

El bloqueo de los receptores adrenérgicos $\alpha 1$, tanto de los clásicos como de los atípicos, aunque en distinta intensidad, se han relacionado con problemas eyaculatorios y con hipotensión ortostática y en ancianos esta última puede provocar inestabilidad y mayor riesgo de insuficiencia cardiaca y de fracturas por caídas ${ }^{4}$.

También se han observado anomalías electrocardiográficas como el alargamiento del intervalo $\mathrm{QT}^{4,5,7}$, constatándose que el sexo femenino es un predictor del fenómeno de alargamiento del QT, ya que las mujeres tienen una probabilidad 3,6 veces mayor de presentar este alargamiento que los hombres ${ }^{12}$ (Tabla 3 ).

En un metaanálisis publicado en 2013 de los estudios en los que se incluyeron pacientes en su primer episodio de esquizofrenia tratados con antipsicóticos de primera o de segunda generación, se observóque los de segunda generación presentaron mayor eficacia que los de primera en la permanencia en el tratamiēnto al tiempo que presentaban menos síntomas extrapiramidales, aunque también constataron mayor aumënto de peso y cambios metabólicos con los de segưnda generación comparado con los de primera ${ }^{14}$.

\section{Antipsicóticos de larga duración}

En los pacientes con esquizofrenia, la falta de adherencia a la medicación es frecuente y en función dê las series oscila entre el 20 y el $89 \%{ }^{15}$. Los antipsicóticos 
de larga duración (ALD) son una opción en el manejo de la esquizofrenia, asumiendo que la eficacia de la molécula es la misma administrada de forma oral que en forma inyectable ${ }^{16}$. Una de las principales ventajas de los ALD para el paciente es no tener que estar pendiente de la toma diaria de la medicación oral, y de esta forma mejorar el cumplimiento, disminuyendo además el riesgo de recaída por abandono del tratamiento ${ }^{16,17}$. Para el médico también significa una ventaja, ya que no deberá estar pendiente de la adherencia al tratamiento, lo cual ayudará a conocer si existen problemas de respuesta al fármaco en caso de no mejoría del cuadro clínico ${ }^{16}$. A pesar de estas ventajas para médico y paciente, los ALD están infrautilizados por muchas razones, como el poco conocimiento sobre ellos por parte del médico, falsas percepciones sobre la eficacia y seguridad de los inyectables, y la percepción negativa que tienen de ellos algunos pacientes ${ }^{17,18}$.

Entre los factores relacionados con el paciente, se ha indicado que la experiencia previa con otros tratamientos inyectables y el tipo de antipsicótico utilizado, la fase clínica de la enfermedad y el tipo de información proporcionada al paciente 0 la ausencia de esta parece que tienen un importante efecto en la aceptación del inyectable ${ }^{19}$. Además, algunos pacientes asocian el inyectable a pérdida de autonomía respecto al tratamiento y mayor estigma de la enfermedad, siendo esta última percepción totalmente inversa en el médico, que considera que causa menos estigma ${ }^{19}$.

Respecto a los posibles factores relacionados con el médico, se ha señalado la falta de experiencia y el mayor coste de la medicación, sin acreditarse de forma objetiva los costes de una politerapia o de una medicación prescrita pero no aplicada, por no hablar de los costes indirectos de un deficitario control de la enfermedad. Por otro lado, la actitud de los psiquiatras respecto a utilizar inyectables es más positiva cuanto mayor experiencia de uso tienen ${ }^{19}$.

Sin embargo, la actitud de los familiares frente a los tratamientos de larga duración, dado que en muchas ocasiones observan la falta de adherencia del paciente al tratamiento, son en general más positivos respecto a su uso ${ }^{19}$. Esto ahonda en la importancia del ambiente familiar para conseguir una buena adherencia al tratamiento ${ }^{19}$.

Una reciente encuesta realizada entre pacientes tratados con antipsicóticos orales o ALD, demostró que los pacientes tratados con ALD preferían esta opción terapéutica y mostraban una importante satisfacción con ella, siendo las principales ventajas comentadas por los pacientes el no tener que recordar diariamente la medicación, no tener que ingerir cada día una pastilla o no tener que estar pendientes de llevar la medicación cuando se viaja ${ }^{18}$.

Un metaanálisis publicado recientemente en el ̨ue se valoraba la eficacia y seguridad de los ALD comparado con los orales indicó que los pacientes tratados con ALD presentaban menor índice de recaída y@menos días de hospitalización ${ }^{20}$.

\section{TRATAMIENTO DE PRIMEROS EPISODIOS}

La prevención de recaídas durante los primeros años de la enfermedad tiene un impacto crítico en los resültados de la esquizofrenia ${ }^{21}$. El retraso en la detección y el tratamiento del primer episodio psicótico se asocia con una recuperación menor y más lenta, así como coñ un riesgo mayor de recaídas ${ }^{22}$, y el adecuado cumplimiênto terapéutico es la variable pronóstica más importânte para la remisión sintomática, la prevención de recaídas y la mejora en la calidad de vida del paciente ${ }^{22}$.

El consenso español sobre la adherencia al tiatamiento de la esquizofrenia indica que el tratamientô de primera línea para mejorar la adherencia terapéutica en pacientes ambulatorios con diagnóstico reciente de psicosis son los ALD de segunda generación ${ }^{23}$. Pö su parte, las guías clínicas de la AFPBN (Association française de psychiatrie biologique et de neurō̄sychopharmacologie) indican que a pesar de que olos antipsicóticos inyectables o de larga duración se han utilizado principalmente en un pequeño grupo despacientes no cumplidores, con recaídas frecuentes oque ponen en riesgo a otras personas, estos ALD están recomendados como tratamiento de mantenimiento después del primer episodio de esquizofrenia ${ }^{24}$.

Los ALD proporcionan al paciente un intervalo- de dosificación más prolongado que las formulaciones orales, y al médico le dan más tiempo para intervènir en caso de problemas de adherencia ${ }^{26}$. De esta forma, Ios ALD presentan beneficios relacionados con la adherencia al tratamiento y a su vez una reduccióno del riesgo de recaída durante las primeras etapas dè la enfermedad o en primeros episodios ${ }^{25}$.

Estas etapas se consideran cruciales en el desarrollo posterior de la enfermedad y existe controversia acêrca de si el adecuado tratamiento de larga duración desde el inicio podría ayudar a modificar el pronóstico y evolución de la enfermedad posteriormente.

Existen estudios que demuestran que los pacientes tratados con ADL están más satisfechos con ellos mismos, con su salud y han mejorado el sueño, indicando además mejor calidad de vida ${ }^{26,27}$. En un estudio de 24 
semanas de duración, se demostró que los pacientes que cambiaron el tratamiento con haloperidol por los ALD mejoraron las funciones cognitivas, incluyendo memoria, función ejecutiva, función motora y atención ${ }^{28}$.

\section{Riesgo de mortalidad de los psicofármacos en la esquizofrenia}

En comparación con los pacientes que presentan cualquier otro trastorno psiquiátrico, los pacientes con esquizofrenia tienen un riesgo de mortalidad muy elevado, y comparado con la población general, su esperanza de vida se acorta en 20-25 años ${ }^{29}$. Un estudio americano realizado de forma retrospectiva con una gran cohorte de pacientes con esquizofrenia de entre 20 y 64 años, reveló que la probabilidad de morir en estos pacientes era 3,5 veces superior a la de la población general, y la principal causa de muerte era por problemas cardiovasculares ${ }^{30}$.

A pesar de que existe controversia sobre la relación de los antipsicóticos con la mortalidad prematura ${ }^{7}$, parece ser que estos fármacos pueden aumentar la mortalidad cardiovascular al aumentar los factores de riesgo como el peso, el desarrollo de diabetes y de dislipemias ${ }^{29}$. Sin embargo, en un análisis de la mortalidad de estudios realizados con pacientes psiquiátricos tratados con psicofármacos, se constató que el riesgo de mortalidad estaba asociado al diagnóstico de la enfermedad (3,8 veces en esquizofrenia, 3,15 veces en depresión y 3 veces en trastorno bipolar), pero este no se aumentaba cuando se trataban farmacológicamente comparado con los pacientes que se trataban con placebo $^{31}$. Por otro lado, una revisión más reciente no obtuvo datos concluyentes para relacionar la medicación antipsicótica con el mayor riesgo de mortalidad ${ }^{29}$.

También se ha valorado el riesgo de mortalidad a corto y largo plazo asociada al uso de antipsicóticos en más de 26.000 pacientes ambulatorios con demencia en una cohorte noruega, comprobando que los antipsicóticos presentan el doble de riesgo de mortalidad que cualquier otro psicofármaco en este tipo de pacientes tanto a corto (30 días) como a largo plazo $(6 \text { años })^{32}$.

\section{Polifarmacia en psiquiatría}

La polifarmacia en psiquiatría es el uso simultáneo de más de un psicofármaco en un mismo paciente, siendo esta práctica muy frecuente ${ }^{33}$. Por otro lado, en esquizofrenia la literatura científica indica que es mejor la monoterapia frente a la combinación de fármacos ${ }^{33}$. Una encuesta realizada entre especialistas sobre el uso de polifarmacia antipsicótica indicó que en más del $23 \%$ de los casos iniciaban con esta polifarmacia y en el $40 \%$ de los casos no intentaron pasar a monoterapia ${ }^{34}$

Una revisión sistemática de 89 estudios en los que se incluía la polifarmacia en pacientes con esquizôfrenia indicó como factores asociados a esta ser joven y varón, inicio temprano de la enfermedad, mayor duración de esta y mayor gravedad o agudeza ${ }^{35}$. Además, los pacientes con polifarmacia psiquiátrica presentaron menor mejoría, mayor resistencia al tratamiento, hisstoria de violencia y más síntomas negativos ${ }^{35}$. La polifarmacia también presenta el riesgo de más efectos secundarios tanto agudos como crónicos, mayor posibilidad de interacciones medicamentosas y mayor posibilidad de incumplimiento terapéutico debido a la complejidad de los distintos regímenes terapéuticos 35 . Además, también se ha asociado la polifarmacia atuna mayor mortalidad en pacientes con esquizofrenia ${ }^{36}$,

Un estudio de cohortes realizado durante más dê 10 años con más de 60.000 pacientes demostró que el tratamiento de la esquizofrenia, aunque sea con polifarmacia, se asocia a un menor riesgo de reingreso hospitalario y de muerte comparado con el no tratamiento ${ }^{37}$. Por tanto, el funcionamiento ideal a la hora de prescribir y utilizar los antipsicóticos sería la mênoterapia, que siempre será preferible a la politeràpia, siendo obviamente más peligroso y deteriorantê el efecto de no tratar.

\section{Principios generales para la prescripción de psicofármacos ${ }^{1,38}$}

Según diversos autores, existen unos principiosabásicos a tener en cuenta para la prescripción dé-un psicofármaco y que se pueden resumir de la siguiênte forma ${ }^{1,39}$ :

- Se debe realizar una valoración completa de lossíntomas del paciente, así como realizar pruebas nêcesarias antes de instaurar un tratamiento con psicofármacos.

- Es necesaria la valoración de los riesgos y benefícios que pueda aportar el tratamiento que se pretende instaurar.

- Evitar siempre que sea posible el uso de combinaciones de fármacos y en caso necesario valoraflas interacciones farmacocinéticas y farmacodinámicas de estos. Se debería también mantener los nivě̉les del fármaco en los rangos terapéuticos ${ }^{40}$.

- Utilizar siempre la dosis mínima eficaz e iniciār o retirar el fármaco de forma gradual. 
Tabla 4. Antipsicóticos inyectables de segunda generación o atípicos

\begin{tabular}{|c|c|c|c|c|}
\hline Fármaco & Indicación & Tecnología utilizada & $\begin{array}{l}\text { Necesidad de dosis } \\
\text { oral inicial }\end{array}$ & Intervalo de dosis \\
\hline Risperidona & $\begin{array}{l}\text { Esquizofrenia, trastorno } \\
\text { bipolar I }\end{array}$ & Microesferas & $\begin{array}{l}\text { Sí, durante } 21 \text { días } \\
\text { de forma simultánea }\end{array}$ & Cada 2 semanas \\
\hline Olanzapina & Esquizofrenia & $\begin{array}{l}\text { Cristales de ácido } \\
\text { pamoico }\end{array}$ & No & Cada 2 o 4 semanas \\
\hline $\begin{array}{l}\text { Paliperidona ( } 1 \text { mes y } 3 \\
\text { meses) }\end{array}$ & Esquizofrenia & Nanocristales & No & $\begin{array}{l}\text { Las } 2 \text { primeras dosis d } \\
\text { formulación } 1 \text { mes se } \\
\text { administrar en } 1 \text { sema }\end{array}$ \\
\hline Aripiprazol monohidrato & $\begin{array}{l}\text { Esquizofrenia, trastorno } \\
\text { bipolar I }\end{array}$ & Monohidrato polimórfico & Sí, 14 días & \\
\hline Aripiprazol lauroxil & Esquizofrenia & Profármaco & Sí, 21 días & Cada 4,6 u 8 semanas \\
\hline
\end{tabular}

- Cuando se cambie de un psicofármaco a otro, valorar la vida media del fármaco que se va a retirar para evitar los síntomas de discontinuación o posibles interacciones.

Para la elección del fármaco se debería tener presente la sintomatología del paciente, el mecanismo de acción del fármaco, su farmacocinética, posibles interacciones, efectos secundarios y patologías concomitantes que presente el paciente ${ }^{38}$. Se deberá tener en cuenta las características de los grupos especiales de pacientes como los ancianos, la población pediátrica y las mujeres embarazadas. Es importante también informar al paciente sobre las ventajas e inconvenientes del tratamiento 38,39 .

\section{Principios generales acerca de la prescripción de antipsicóticos de larga duración}

Las diferencias entre los distintos ALD se pueden observar en la tabla 4.

Existen estudios realizados con el fin de evaluar la funcionalidad de los pacientes tratados con los ALD. En uno de ellos se incluyeron pacientes hospitalizados y ambulatorios con trastornos psicoafectivos que presentaban una exacerbación aguda. En el estudio se evaluaron los dominios específicos de funcionalidad en la escala Personal and Social Performance (PSP). Los pacientes fueron tratados durante 25 semanas en diseño abierto, con palmitato de paliperidona inyectable una vez al mes en forma de monoterapia o combinado con antidepresivos o estabilizantes del humor. Los pacientes que alcanzaron la estabilización se asignaron aleatoriamente en forma de doble ciego a recibir tratamiento con paliperidona o con placebo durante $15 \mathrm{me}$ ses. Los resultados mostraron mejoría de todos los dominios de la escala PSP en los pacientes tratälos con paliperidona, tanto en el periodo abierto come en el de doble ciego, comparado con el descenso observado en los pacientes tratados con placebo, indicando que los pacientes tratados con paliperidona mantưvieron su funcionalidad ${ }^{41}$.

Más recientemente, otro estudio, esta vez con apipiprazol lauroxil de larga duración frente a placebo, y en el que se utilizaron las escalas Positive and Negátive Syndrome Scale (PANSS) y PSP, también demosstró mejoría en la puntuación de ambas (de forma estadísticamente significativa) en los pacientes tratados con aripiprazol comparados con los pacientes tratados con placebo $^{42}$.

\section{Criterios de calidad en esquizofrenia, depresión y trastorno bipolar ${ }^{43}$}

En el año 2015, la Fundación Española de Psiqüilatría, la Sociedad Española de Psiquiatría y la Sociedad Española de Psiquiatría Biológica, junto con la Sơciedad Española de Calidad Asistencial, publicaron ùnos criterios/indicadores de calidad de la asistencia délos pacientes con esquizofrenia, depresión y trastorno bipolar en nuestro país. Los principales indicadores de resultados que reflejan la calidad asistencial en eștas tres patologías se resumen en:

- Idoneidad del ingreso en la unidad de agudos ynúmero de reingresos hospitalarios.

- Recurrencia de un nuevo episodio del trastorno mental después de haberse recuperado de otro $(6$ meses libre de síntomas). El riesgo de recurrencia en depresión mayor (DM) es del 50\% después del primer episodio y aumenta tras los siguientes, mientras que en la psicosis, el 30-50\% de los pacientes sufren una recurrencia durante el primer año después del primer 
episodio. Por su parte, el trastorno bipolar (TB) es un trastorno recurrente. En las tres patologías, el principal factor de riesgo para la recurrencia es la interrupción/supresión del tratamiento.

- Índice de suicidios y conducta suicida. La DM se asocia con la conducta suicida, y el riesgo de suicidio es mayor durante el episodio y en el periodo de remisión parcial. En pacientes con esquizofrenia la tasa de suicidios es del 10\% y está relacionada con la presencia de alucinaciones y depresión. En el TB el riesgo de suicidio es mayor cuando se da un viraje rápido de manía o hipomanía a DM.

- Aparición de efectos secundarios como la disfunción sexual, efectos cardiovasculares o metabólicos.

- Daños físicos (caídas), frecuente en las residencias y centros sociosanitarios.

- Programas psicoeducativos para pacientes.

- Adherencia al tratamiento y tasa de abandonos. La falta de adherencia en DM puede llegar hasta el 50$60 \%$ de los pacientes debido principalmente al propio trastorno y los efectos secundarios de la medicación. En esquizofrenia y en TB, con porcentajes similares, los principales motivos son los efectos secundarios y la baja conciencia de enfermedad.

- Abuso de sustancias tóxicas, que es más prevalente en estos pacientes que en la población general.

- Necesidad de contención mecánica. La prevalencia de la contención mecánica es de entre el 8 y el $24 \%$ en pacientes atendidos en Servicios de Urgencias y cerca del $22 \%$ en pacientes atendidos en Servicios de Psiquiatría.

\section{Conclusiones}

Por su eficacia demostrada, los psicofármacos son el tratamiento de elección para las patologías psiquiátricas. Antes de la elección de un psicofármaco se deberá valorar cuidadosamente al paciente, así como las características del fármaco elegido y el beneficio-riesgo de su administración.

Los fármacos antipsicóticos de ambas clases, primera o segunda generación, nos pueden aportar eficacias similares sobre sintomatología positiva. No obstante, existe una diferencia sustancial en los efectos secundarios y en la mejora sobre la sintomatología negativa a favor de los antipsicóticos de segunda generación.

Entre los antipsicóticos de segunda generación y atendiendo a su perfil receptor, las diferencias también son significativas en cuanto a sus efectos secundarios, debiendo valorar en cada caso qué perfil es adecuado.
No obstante las peculiaridades de la patología tratada, la escasa adherencia al tratamiento por ausencia de insight hacen que en nuestras variables de decisión adquieran mayor importancia la aparición de los $\mathrm{A} L \mathrm{~L}$ y entre ellos los de segunda generación, que cômo hemos dicho aportan una diferencia sustancial.

Tratar los primeros episodios de una manera adecuada utilizando dosis bajas y fármacos lo menos: incapacitantes posibles, pero a su vez asegurando el tratamiento con la vía de administración inyectable parece ser el modo más seguro de evitar futuras recaidas e intentar paliar el deterioro que la aparición de estas conllevan para el paciente.

Encontrar el equilibrio para tratar adecuadamente y no abusar de la politerapia más que en las situaciönes especialmente complejas es esencial también parâ reducir la mortalidad de nuestros pacientes.

Finalmente, toda esta manera de proceder es conveniente y viene a reforzar los criterios de calidad establecidos en la unidad de agudos, mejorando a la lărga la funcionalidad de nuestros pacientes, su evoluciốn y posible pronóstico, que van a depender en mayôr 0 menor medida de nuestras decisiones como clínicōs.

\section{Responsabilidades éticas}

Protección de personas y animales. Los autöres declaran que para esta investigación no se han realizado experimentos en seres humanos ni en animales.

Confidencialidad de los datos. Los autores declaran que han seguido los protocolos de su centro-de trabajo sobre la publicación de datos de pacientes.

Derecho a la privacidad y consentimiento informado. Los autores declaran que en este artículö no aparecen datos de pacientes.

\section{Bibliografía}

1. The European Mental Health Action Plan 2013-2020 [Internet]. Gïébra: World Health Organization; 2015 [último acceso: sept 2019]. Disponible en: http://www.euro.who.int/ data/assets/pdf file/0020/280604/WHOEurope-Mental-Health-Acion-Plan-2013-2020.pdf

2. Basterra V. Evolución de la prevalencia de alto riesgo de trastornos mentales en población adulta española: 2006-2012. Gac Sanit. 2017;31(4):324-6

3. Braslow JT, Marder SR. History of psychopharmacology. Annu Rev Clin Psychol. 2019;15:25-50.

4. Alamo C, Cuenca E, López-Muños F. Avances en psicofarmacología y perspectivas de futuro [Internet]. Madrid, España; 2004: Real Academia Nacional de Farmacia, Monografías XV, Nuevos avances en medicamentos [último acceso: sept 2019]. Disponible en: http://www.analesrant.com/ index.php/mono/article/viewFile/539/557\&a=bi\&pagenumber $=1 \& w=100$

5. Bohorquez Peñaranda A, Gómez Restrepo C, García Valencia J, Jaramillo González LE, de la Hoz AM, Arenas A, et al. Tratamiento con antipsicóticos en la fase aguda del paciente adulto con diagnóstieo de esquizofrenia. Rev Colomb Psiquat. 2014;43(S1):13-28.

6. Arenas Borrero A, Gómez Restrepo C, Bohórquez Peñaranda AP, Vélez Traslaviña A, Castro Díaz SM, Jaramillo González LE, et al. Prevención y tratamiento de los efectos adversos agudos más frecuentes por el uso de antipsicóticos en pacientes adultos con diagnóstico de esquizofrenia. Rev Colomb Psiquat. 2014;43(S1):66-74. 
7. Correll CU, Rubio JM, Kane JM. What is the risk-benefit ratio of long-term antipsychotic treatment in people with schizophrenia? World Psychiatry. 2018;17(2):149-60.

8. Gründer G, Heinze M, Cordes J, Mühlbauer B, Juckel G, Schulz C, et al. Effects of first-generation antipsychotics versus second-generation antipsychotics on quality of life in schizophrenia: a double-blind, randomised study. Lancet Psychiatry. 2016;3(8):717-29.

9. Carbon M, Kane JM, Leucht S, Correll CU. Tardive dyskinesia risk with first- and second-generation antipsychotics in comparative randomized controlled trials: a meta-analysis. World Psychiatry. 2018;17(3):330-40.

10. Pérez Escudero A, Gil Molina S, Pina Camacho L, García-Cabeza I Psicofarmacología de la psicosis: elección del fármaco, adherencia al tratamiento y nuevos horizontes. Clin Salud. 2010;21(3):271-83.

11. Landa Contreras E, Fortes Álvarez JL. Monoterapia versus politerapia en el tratamiento de la psicosis: ¿con qué nos quedamos? Revista Clínica de Medicina de Familia. 2011;4(2):140-5.

12. Sáez de Adana García de Acilua E, Cano Al, Eizaguirre García A, López Zurbano S, Francos Ajona F, Gaviña Arenaza J, et al. Efectos adversos de antipsicóticos atípicos, diferencias según sexo. Psiq Biol. 2014;21(3):95-101.

13. Kessing LV, Thomsen AF, Mogensen UB, Andersen PK. Treatment with antipsychotics and the risk of diabetes in clinical practice. Br J Psychiatry. 2010;197(4):266-71.

14. Zhang JP, Gallego JA, Robinson DG, Malhotra AK, Kane JM, Correll CU. Efficacy and safety of individual second-generation vs. first-generation antipsychotics in first-episode psychosis: a systematic review and meta-analysis. Int J Neuropsychopharmacol. 2013;16(6):1205-18.

15. Phan SV. Medication adherence in patients with schizophrenia. Int Psychiatry Med. 2016:51(2):211-9.

16. Citrome $L$. Long-acting injectable antipsychotics update: lengthening the dosing interval and expanding the diagnostic indications. Expert Rev Neurother. 2017;10:1029-43

17. Correll CU, Citrome L, Haddad PM, Lauriello J, Olfson M, Calloway SM, et al. The use of long acting injectable antipsychotics in schizophrenia: Evaluating the evidence. J Clin Psychiatry. 2016;77(suppl3):1-24.

18. Mace S, Chak O, Punny S, Sedough-Abbasian D, Vegad C, Taylor DM Positive views on antipsychotic long-acting injections: results of a survey of community patients prescribed antipsychotics. Ther Adv Psychopharmacol. 2019;9:2045125319860977.

19. Arango C, Baeza I, Bernardo M, Cañas F, de Dios C, Diaz-Marsá M, et al. Antipsicóticos inyectables de liberación prolongada para el tratamiento de la esquizofrenia en España. Rev Psiquiatr Salud Ment. 2019;12(2):92-105

20. Park SC, Choi MY, Choi J, Park E, Tchoe HJ, Suh JK, et al. Comparative efficacy and safety of long-acting injectable and oral second-generation antipsychotics for the treatment of schizophrenia: A systematic review and meta-analysis. Clin Psychopharmacol Neurosci. 2018;16(4):361-75.

21. Caseiro O, Pérez-Iglesias R, Mata I, Martínez-Garcia O, Pelayo-Terán JM, Tabares-Seisdedos R, et al. Predicting relapse after a first episode of non-affective psychosis: a three-year follow-up study. J Psychiatr Res. 2012;46(8):1099-105.

22. Arango $\mathrm{C}$, Bernardo $\mathrm{M}$, Bonet $\mathrm{P}$, Cabrera A, Crespo-Facorro $\mathrm{B}$, Cuesta MJ, et al. Cuando la asistencia no sigue a la evidencia: el caso de la falta de programas de intervención temprana en psicosis en España. Rev Psiquiatr Salud Ment. 2017:10(2):78-86.

23. Roca M, Cañas F, Olivares J, Rodríguez A, Giner J. Treatment adherence in schizophrenia. Spanish clinical consensus. Actas Esp Psiquiatr 2007;35(1 Suppl):1-6.

24. Llorca PM, Abbar M, Courtet P, Guillaume S, Lancrenon S, Samalin L. Guidelines for the use and management of long-acting injectable antipsychotics in serious mental illness. BMC Psychiatry. 2013;13:340.

25. Miyamoto S, Wolfgang Fleischhacker W. The use of long-acting injectable antipsychotics in schizophrenia. Curr Treat Options Psychiatry. 2017;4(2):117-26.
26. Marinis T, Saleem P, Glue P, Arnoldussen W, Teijeiro R, Lex A, et al. Switching to long-acting injectable risperidone is beneficial with regard to clinical outcomes, regardless of previous conventional medication in patients with schizophrenia. Pharmacopsychiatry. 2007;40:257-63. N

27. Mihajlovic G, Jovanovic-Mihajlovic N, Radmanovic B, Radonjic K K Djukic-Dejanovic S, Jankovic S, et al. Quality of life of schizophrenic patients treated with haloperidol depot and injection preparation of long-lasting risperidone. Srp Arh Celok Lek. 2011;139:36-40.

28. Suzuki H, Gen K. The influence of switching from haloperidol decăboate depot to risperidone long-acting injection on the clinical symptoms and cognitive function in schizophrenia. Hum Psychopharmacol. 2012;27: 470-5.

29. Vermeulen J, van Rooijen G, Doedens $P$, Numminen E, van Tricht $\bar{M}$, de Haan L. Antipsychotic medication and long-term mortality risk in patients with schizophrenia; a systematic review and meta-analysis. PsychollMed. 2017;47(13):2217-28.

30. Olfson M, Gerhard T, Huang C, Crystal S, Stroup TS. Premature mortality among adults with schizophrenia in the United States. JAMA Psyehiatry. 2015;72(12):1172-81.

31. Khan A, Faucett J, Morrison S, Brown WA. Comparative mortality fisk in adult patients with schizophrenia, depression, bipolar disorder, anxiety disorders, and attention-deficit/hyperactivity disorder participating in psychopharmacology clinical trials. JAMA Psychiatry. 2013;70(10):1091-9.

32. Langballe EM, Engdahl B, Nordeng H, Ballard C, Aarsland D, Selbæk G. Short- and long-term mortality risk associated with the use of antipsychotics among 26,940 dementia outpatients: a population-based study. Am J Geriatr Psychiatry. 2014;22(4):321-31.

33. Stucchi-Portocarrero S, Saavedra J.E. Polifarmacia psiquiátrica efpersonas con esquizofrenia en un establecimiento público de salud "mental en Lima. Rev Neuropsiquiatr. 2018;81(3):145-53.

34. Correll CU, Shaikh L, Gallego JA, Nachbar J, Olshanskiy V, Kishimoto T, et al. Antipsychotic polypharmacy: a survey study of prescriber attifüdes, knowledge and behavior. Schizophr Res. 2011:131(1-3):58-62.

35. Correll CU, Gallego JA. Antipsychotic polypharmacy: a comprehensive evaluation of relevant correlates of a long-standing clinical practice-Psychiatr Clin North Am. 2012;35(3):661-81.

36. Auquier $\mathrm{P}$, Lançon $\mathrm{C}$, Rouillon $\mathrm{F}$, Lader M, Holmes C. Mortality ín-schizophrenia. Pharmacoepidemiol Drug Saf. 2006;15(12):873-9.

37. Tiihonen J, Taipale $H$, Mehtälä J, Vattulainen $P$, Correll CU, Tanskanen A Association of antipsychotic polypharmacy vs monotherapy with psychiatric rehospitalization among adults with schizophrenia. JAMA Psychiatry. 2019;76(5):499-507.

38. Bravo-Ortiz MF. Psicofarmacología para psicólogos. Madrid, Esp̧aña: Editorial Síntesis; 2003.

39. NHS guidelines. Guidance on the use of antipsychotics. Version 4 Iinternet]. Reino Unido: NHS; April 2018. Disponible en: https://www.sussexpartnership.nhs.uk/sites/default/files/documents/antipsychotid_güdelines_v4_-_apr_2018_-_final_3.pdf

40. Silva H. Psychopharmacology and medical pathology. Rev Med Clin Condes. 2017;28(6):830-4.

41. Fu DJ, Turkoz I, Walling D, Lindenmayer JP, Schooler NR, Alphs L. Paliperidone palmitate once-monthly maintains improvement in fumctioning domains of the Personal and Social Performance scale compared with placebo in subjects with schizoaffective disorder. SchizophrRes. 2018;192:185-93

42. Correll CU, Stanford AD, Claxton A, Du Y, Weiden PJ. Social and functional outcomes with two doses of aripiprazole lauroxil vs placebo in patients with schizophrenia: a post-hoc analysis of a 12-week phase 3 efficacy study. Psychiatry Res. 2019;274:176-81.

43. Roca Bennasar M, Garcia El. Criterios de calidad en psiquiatría. España: Editorial Luzán 5; 2016. 